\title{
APPROXIMATION OF VECTOR-VALUED CONTINUOUS FUNCTIONS ${ }^{1}$
}

\author{
ALAN H. SHUCHAT
}

\begin{abstract}
The results of this article are important for proving Riesz-type representation theorems for spaces of continuous functions with values in a topological vector space. It is well known that every continuous function with compact support from a locally compact Hausdorff space to a locally convex space can be uniformly approximated by continuous functions with finite-dimensional range. We give several conditions sufficient for this to be true without convexity. This problem is related to a vector-valued Tietze extension problem, and we give a new proof of a theorem of Dugundji, Arens, and Michael in this area, using topological tensor products.
\end{abstract}

Let $T$ be a compact Hausdorff space, $E$ a topological vector space (TVS) over either the real or complex field, and $C(T, E)$ the space of continuous functions from $T$ to $E$, with the topology of uniform convergence. When $E$ is the scalar field we write $C(T)$ instead of $C(T, E)$. For each $a \in C(T)$ and $x \in E$ the function $t \rightarrow a(t) x$ from $T$ to $E$, denoted by $a \otimes x$, is continuous. The linear span of these functions in $C(T, E)$ is the set of all finite sums $\sum a_{i} \otimes x_{i}$ with $a_{i} \in C(T)$ and $x_{i} \in E$ and is isomorphic to the algebraic tensor product $C(T) \otimes E$. If $E$ is locally convex, then $C(T) \otimes E$ is dense in $C(T, E)$ [4, Chapter III, $\S 1$, Proposition 1 and Lemma 2]. This property, the density property, is of prime importance in the representation of linear functionals and operators on $C(T, E)$ by vector measures [12]. The sufficient conditions given in this article have been used by the author [11] to extend these representations to the case when $E$ is not assumed to be locally convex. It is not known if any space $C(T, E)$ fails to have the density property.

Presented to the Society, November 29, 1969; received by the editors December 31, 1969 and, in revised form, April 23, 1971.

AMS 1969 subject classifications. Primary 4160, 4625; Secondary 2850, 5460.

Key words and phrases. Topological vector spaces, vector-valued continuous functions, vector measures, extension of vector-valued functions.

${ }^{1}$ Most of these results appeared in the author's doctoral dissertation at the University of Michigan, directed by Professor M. S. Ramanujan. The author wishes to thank Professor Ramanujan, for his friendly advice and encouragement, as well as Professor Joel $\mathbf{H}$. Shapiro and the referee, for certain valuable suggestions. 
The results of this article extend to locally compact Hausdorff spaces. If $X$ is such a space, let $C(X, E)$ denote the space of continuous functions from $X$ to $E$ with compact support, with the topology of uniform convergence. Then $C(X) \otimes E$ is dense in $C(X, E)$ if $C(T, E)$ has the density property for every compact subspace $T \subset X$.

If $f=\sum^{n} a_{i} \otimes x_{i} \in C(T) \otimes E$, then $f$ has finite-dimensional range. When $E$ is a Hausdorff space this property characterizes $C(T) \otimes E$.

Proposition 1. If $E$ is a Hausdorff space, then $C(T) \otimes E$ consists of all functions in $C(T, E)$ having finite-dimensional range.

Proof. If $f \in C(T, E)$ and $\left\{x_{1}, \cdots, x_{n}\right\}$ is a basis for $f(T)$, then $f(T)$ is topologically isomorphic to the product of $n$ copies of the scalar field. The coefficient functionals $\lambda_{i}$ on $f(T)$ defined by $f(t)=\sum \lambda_{i}[f(t)] x_{i}$ are continuous, each $a_{i}=\lambda_{i} \cdot f$ is continuous and $f=\sum a_{i} \otimes x_{i}$.

The following theorem is contained in a result of Turpin and Waelbroeck [14] on differentiable functions but our proof is much simpler for this special case. We generalize the usual proof of the density property in locally convex spaces to cover the case when $T$ has finite covering dimension [10]. If $\gamma$ is a covering of a topological space $X$, the order of $\gamma$ is

$$
\sup _{x \in X} \sup \{n: x \text { belongs to } n \text { members of } \gamma\} \text {. }
$$

THEOREM 1. If $T$ has finite covering dimension and $E$ is a TVS, then $C(T, E)$ has the density property.

Proof. Let $n$ be the dimension of $T, f \in C(T, E)$ and $U$ an open balanced 0-neighborhood in $E$. We will produce a function $g \in C(T) \otimes E$ such that $g-f$ maps $T$ into $U$. Each $t \in T$ lies in the open set $V_{t}=$ $f^{-1}\{f(t)+U\}$ and a finite number of these sets, say $V_{1}, \cdots, V_{m}$, suffice to cover $T$.

Let $a_{1}, \cdots, a_{m}$ be a continuous partition of unity subordinate to this covering. Let $t_{i} \in V_{i}$ and $g=\sum_{i=1}^{m} a_{i} \otimes f\left(t_{i}\right)$. Then $g \in C(T) \otimes E$ and

$$
g(t)-f(t)=\sum_{i=1}^{m} a_{i}(t)\left[f\left(t_{i}\right)-f(t)\right] .
$$

If $t \in V_{i}$, then $f\left(t_{i}\right)-f(t) \in U$ and if $t \notin V_{i}$, then $a_{i}(t)\left[f\left(t_{i}\right)-f(t)\right]=0$.

Since $U$ is balanced, each $g(t)-f(t)$ belongs to the $k$-fold sum $U+\cdots+U$, where $k$ is the number of sets $V_{i}$ containing $t$. Every open covering of $T$ has a refinement of order at most $n+1$ that is also an open covering $[10$, pp. 9, 97] and since $T$ is compact we may assume that the refinement is finite. If we take such a refinement of the covering $\left\{V_{t}\right\}$ and define a partition of unity $\left(a_{i}\right)$ subordinate to this refinement, then 
$g(t)-f(t)$ belongs to the $(n+1)$-fold sum $U+\cdots+U$ for each $t \in T$. Since $n$ depends only on $T$, the proof is complete.

We note that if $U$ is convex, then by (1), $g(t)-f(t) \in U$ for all $q$, so when $E$ is locally convex $C(T, E)$ has the density property regardless of the dimension of $T$.

Theorem 1 extends to compact spaces with an approximation-type property based on the uniform structure.

COROLLARY 1. If the identity map on $T$ can be uniformly approximated by continuous maps with finite-dimensional range, then $C(T, E)$ has the density property for each TVS E.

Proof. Let $\left(u_{\alpha}\right)$ approximate the identity, $T_{\alpha}$ be the quotient of $T$ by the relation $\left\{(s, t): u_{\alpha}(s)=u_{\alpha}(t)\right\}, \pi_{\alpha}$ the quotient map from $T$ to $T_{\alpha}$, $f \in C(T, E)$, and $f_{\alpha} \in C\left(T_{\alpha}, E\right)$ the map induced by $f \cdot u_{\alpha}$. Since $T$ is compact, $T_{\alpha}$ is homeomorphic to $u_{\alpha} T$ and all the maps in question are uniformly continuous, so $\left(f \cdot u_{\alpha}\right)$ converges uniformly to $f$. Also, each $f_{\alpha}$ is the uniform limit of functions in $C\left(T_{\alpha}\right) \otimes E$ and the maps from $T$ to $E$ induced by these functions belong to $C(T) \otimes E$ and converge uniformly to $f \cdot u_{\alpha}$.

Corollary 2. Let $T$ be imbedded in a cube $K$. If each $f \in C(T, E)$ has a continuous extension $f^{\prime}$ to $K$, then $C(T, E)$ has the density property.

Proof. The identity map on a cube can be approximated uniformly by projections onto products of finitely many factors. Thus $f^{\prime}$ can be approximated by mappings in $C(K) \otimes E$ and their restrictions to $T$ yield the desired result.

Extensions of this type have been studied by several authors. In certain cases where $E$ is locally convex (and so $C(T, E)$ already has the density property), Arens [1, p. 15] and Dugundji [5, p. 37] have obtained extensions. For the general case, we make two definitions following Klee [7], [8]. Let $\boldsymbol{C}$ be the class of all compact Hausdorff spaces. We say $E$ is admissible if $C(T, E)$ has the density property for all $T \in C$ (this is equivalent to Klee's definition) and is an extension space for $C$ if for all $K \in C$, every continuous map from a closed subset $T$ of $K$ into $E$ has a continuous extension to $K$. Then we have the following corollary.

COROllary 3. Every extension space for $\boldsymbol{C}$ is admissible.

Recall that an $F$-space is a complete metrizable TVS. The next result is an immediate consequence of Corollary 3 and a theorem of Klee [7, p. 284].

COROllary 4. An F-space is admissible if and only if it is an extension space for $\boldsymbol{C}$. 
For locally convex spaces, topological tensor products and the density property can be used to prove "simultaneous" extension theorems for vector-valued functions directly from the corresponding theorems for real-valued functions. We illustrate this in the case of Corollary 2. The spaces involved are over the real field. The result is known [9, p. 802], but the present method simplifies the proof of the vector-valued case. If $K$ is metrizable, then the map $B$ below may be taken from Borsuk's theorem [3] and Arens' more complex result [1] need not be considered.

THEOREM 2. Let T be a closed metric subspace of a compact Hausdorff space $K$. If $E$ is a complete locally convex Hausdorff space, there is a topological isomorphism $A$ of $C(T, E)$ onto a subspace of $C(K, E)$ that is an extension mapping, i.e., Af is an extension of $f$ for every $f \in C(T, E)$.

Proof. There is an isometric isomorphism $B$ of $C(T)$ onto a subspace of $C(K)$ that is an extension mapping [1, p. 20]. Also, $C(T, E)$ can be identified with $C(T) \hat{\otimes}_{\epsilon} E$, the completion of the algebraic tensor product for the topology $\otimes_{\epsilon}$ of bi-equicontinuous convergence, and $C(S, E)$ with $C(S) \hat{\otimes}_{\epsilon} E[6$, p. 90]. The map $B \otimes I$, where $I$ is the identity map on $E$, is a topological isomorphism and its extension $A$ to $C(T, E)$ answers our requirements [13, p. 440].

When $E$ is not locally convex $\otimes_{\epsilon}$ is no longer appropriate, but if $E$ is a complete $p$-normed space, there is a topology for $C(T) \otimes E$ that is reminiscent of $\otimes_{\epsilon}$. In fact, $C(T, E)$, as a complete $p$-normed space, is topologically isomorphic to a subspace of the $p$-normed space $L(M(T), E)$. Here (see [11] for the integration theory) $M(T)$ is the $p$-normed space of finite regular Borel measures on $T$ that are of bounded variation as $L(E, E)$-valued measures, i.e., the $p$-norm

$$
\operatorname{Var} \mu=\sup \sum\left|\mu\left(A_{i}\right)\right|^{p},
$$

taken over all finite partitions of $T$ by Borel sets, is finite, and the isomorphism $J$ is given by $J f(\mu)=\int f d \mu$. However, we have not been able to show that the map $B \otimes I$ is an isomorphism.

Another class of conditions under which $C(T, E)$ has the density property involves restrictions on $E$.

THEOREM 3. Suppose the identity map on $E$ can be approximated uniformly on precompact sets by continuous maps, where the range of each map has finite covering dimension. Then $E$ is admissible.

Proof. If $T \in C, f \in C(T, E)$ and $U$ is a 0 -neighborhood in $E$, there is a continuous map $u$ on $f T$ whose range $S$ has finite covering dimension and $u x-x \in U$ for all $x \in f T$. By Theorem 1, there is a map $v \in C(S) \otimes E$ such that $v s-s \in U$ for all $s \in S$. Then $g=v \cdot u \cdot f \in C(T) \otimes E$ and $g-f$ 
maps $T$ into $U+U$. So $C(T, E)$ has the density property and $E$ is admissible.

We define the approximation property for $E$ just as for locally convex spaces, i.e., the maps in Theorem 3 are linear and have range in a finitedimensional linear subspace of $E$.

COROLLARY 1. If E has the approximation property, then $E$ is admissible.

In an $F$-space, the "norm" $\|x\|=d(x, 0)$ defined by the metric $d$ is called an $F$-norm. We assume $d$ is translation-invariant and has balanced 0 -spheres.

COROllary 2 [8, p. 294]. If $E$ is an F-space with a basis, then $E$ is admissible.

Proof. By a theorem of Arsove [2] the coefficient functionals $F_{n}$ of a basis $\left(e_{n}\right)$ in $E$ are continuous, i.e., the basis is a Schauder basis. Applying the Banach-Steinhaus theorem to the maps $x \rightarrow \sum^{N} F_{n}(x) e_{n}$, we see that $E$ has the approximation property.

COROLlARY 3. $C\left(T, l^{p}\right)$ has the density property for all $p>0$.

We extend this result to certain nonmetrizable spaces with bases. The topology of every TVS is determined by pseudo-metrics in the sense that their 0-spheres form a local subbase. It is clear that if $E$ has a determining family of complete metrics such that $E$ has a basis with respect to each one, then we can carry out the proof of Corollary 2 for each metric to show that $E$ is admissible. One way in which this situation arises is the following.

Let $E$ be a TVS with a basis $\left(e_{n}\right)$ and coefficient functionals $F_{n}$, which need not be continuous. Let $E$ have a determining family of complete metrics and $P$ be the corresponding family of $F$-norms. For each $p \in P$, let $E_{p}$ denote $E$ with the $p$-topology. Then $\left(e_{n}\right)$ need not be a basis for $E_{p}$, since the expansion of an element is unique in $E$ and converges in $E_{p}$ but may not be unique in $E_{p}$. In Theorem 4 we give a sufficient (and clearly necessary) condition for uniqueness.

THEOREM 4. If for some $p \in P, x=\sum h_{n} e_{n}$ in $E_{p}$ implies

$$
\sup _{m} p\left[\sum^{m} F_{n}(x) e_{n}\right] \leqq \sup _{m} p\left[\sum^{m} h_{n} e_{n}\right],
$$

then $\left(e_{n}\right)$ is a Schauder basis for $E_{p}$ and $E$. Thus if this is true for each $p \in P$, then $E$ is admissible.

Proof. We use techniques from [2]. The functional

$$
p^{\prime}(x)=\sup _{m} p\left[\sum^{m} F_{n}(x) e_{n}\right]
$$


is well defined and determines a translation-invariant metric linear topology on $E$. $E_{p^{\prime}}$, which denotes $E$ with the $p^{\prime}$-topology, is stronger than $E_{p}$ since $p^{\prime}$ dominates $p$. We show that in any case, $\left(e_{n}\right)$ is a Schauder basis for $E_{p}$, with the same coefficient functionals as for $E$. For every $N$,

$$
p^{\prime}\left[x-\sum_{n=1}^{N} F_{n}(x) e_{n}\right]=\sup _{m} p\left[\sum_{n=N+1}^{m} F_{n}(x) e_{n}\right],
$$

so that $\sum F_{n}(x) e_{n}$ converges to $x$ in $E_{p^{\prime}}$. The representation is unique because if $\sum h_{n} e_{n}=0$ in $E_{p^{\prime}}$ then, for every $N$,

$$
\lim _{m \rightarrow \infty} F_{N}\left(\sum^{m} h_{n} e_{n}\right)=0 .
$$

But $F_{N}\left(\sum^{m} h_{n} e_{n}\right)=h_{N}$ for all $m \geqq N$, so the coefficients are all zero. Since

$$
p^{\prime}\left[F_{n}(x) e_{n}\right]=p\left[F_{n}(x) e_{n}\right] \leqq 2 p^{\prime}(x)
$$

and $e_{n} \neq 0$, each $F_{n}$ is continuous on $E_{p^{\prime}}$.

In order to complete the proof it suffices to show that $E_{p}=E_{p^{\prime}}$, i.e., the identity map on $E$ is a homeomorphism for $p$ and $p^{\prime}$. The set $S$ of scalar sequences $s=\left(h_{n}\right)$ such that $\sum h_{n} e_{n}$ converges in $E_{p}$ is a vector space and the sequences $x^{*}=\left(F_{n}(x)\right)$ form a subspace $S^{\prime}$. It is not difficult to show that $q(s)=\sup _{m} p\left[\sum^{m} h_{n} e_{n}\right]$ is an $F$-norm for an $F$-space topology on $S$ and that $x^{*} \leftrightarrow x$ is an isomorphism between $S^{\prime}$ and $E_{p^{\prime}}$. $S^{\prime}$ and the subspace $S^{\prime \prime}$ of sequences $\left(h_{n}\right)$ for which $\sum h_{n} e_{n}=0$ are algebraically complementary.

Let $s=\left(h_{n}\right) \in S, x=\sum h_{n} e_{n}$ and $[s]$ be the coset in $S / S^{\prime \prime}$ corresponding to $s$. The projection $s \rightarrow x^{*}$ of $S$ onto $S^{\prime}$ is continuous since, by hypothesis, $q\left(x^{*}\right)=p^{\prime}(x) \leqq q(s)$. Thus $S^{\prime}$ and $S^{\prime \prime}$ are topological complements and $[s] \leftrightarrow x$ is an isomorphism between $S / S^{\prime \prime}$ and $E_{p^{\prime}}$. The mapping $s \rightarrow x$ from $S$ onto $E_{p}$ is linear and continuous, since $p(x) \leqq$ $p^{\prime}(x) \leqq q(s)$, and its kernel is $S^{\prime \prime}$. By the open mapping theorem, $[s] \leftrightarrow x$ is then an isomorphism between $S / S^{\prime \prime}$ and $E_{p}$ and the proof is complete.

\section{REFERENCES}

1. R. F. Arens, Extension of functions on fully normal spaces, Pacific J. Math. 2 (1952), 11-22. MR 14, 191.

2. M. G. Arsove, The Paley-Wiener theorem in metric linear spaces, Pacific J. Math. 10 (1960), 365-379. MR 23 \#A2731.

3. K. Borsuk, Isomorphie der Funktionalräume, Bull. Acad. Polon. Sci. Ser. A (1933), $1-10$.

4. N. Bourbaki, Intégration. Chaps. I-IV, 2ième éd., Actualités Sci. Indust., no. 1175, Hermann, Paris, 1965. MR 36 \#2763.

5. J. Dugundji, An extension of Tietze's theorem, Pacific J. Math. 1 (1951), 353-367. MR 13, 373. 
6. A. Grothendieck, Produits tensoriels topologiques et espaces nucléaires, Mem. Amer. Math. Soc. No. 16 (1955). MR 17, 765.

7. V. Klee, Shrinkable neighborhoods in Hausdorff linear spaces, Math. Ann. 141 (1960), 281-285. MR 24 \#A1003

8. - Leray-Schauder theory without local convexity, Math. Ann. 141 (1960), 286-296. MR 24 \#A1004.

9. E. A. Michael, Some extension theorems for continuous functions, Pacific J. Math. 3 (1953), 789-806. MR 15, 547.

10. J. Nagata, Modern dimension theory, Bibliotheca Math., vol. 6, Interscience, New York, 1965. MR \#8380.

11. A. H. Shuchat, Integral representation theorems in topological vector spaces (to appear).

12. K. Swong, A representation theory of continuous linear maps, Math. Ann. 155 (1964), 270-291; errata, ibid. 157 (1964), 178. MR 29 \#2642.

13. F. Treves, Topological vector spaces, distributions and kernels, Academic Press, New York, 1967. MR 37 \#726.

14. P. Turpin and L. Waelbroeck, Sur l'approximation des fonctions différentiables à valeurs dans les espaces vectoriels topologiques, C. R. Acad. Sci. Paris Sér. A-B 267 (1968), A94-A97. MR 38 \#2597.

Department of Mathematics, University of Toledo, Toledo, Ohio 43606

Current address: Mount Holyoke College, South Hadley, Massachusetts 01075 